DOI: http://dx.doi.org/10.21123/bsj.2018.15.4.0472

\title{
On Preradical of Semimodules
}

\author{
Sarah H. A. Alsaebari*
}

Asaad M. A. Alhossaini

Received 15/5/2018, Accepted 2/10/2018, Published 6/12/2018

This work is licensed under a Creative Commons Attribution 4.0 International License.

\begin{abstract}
:
In this paper we study the notion of preradical on some subcategories of the category of semimodules and homomorphisms of semimodules.

Since some of the known preradicals on modules fail to satisfy the conditions of preradicals, if the category of modules was extended to semimodules, it is necessary to investigate some subcategories of semimodules, like the category of subtractive semimodules with homomorphisms and the category of subtractive semimodules with ek-regular homomorphisms.
\end{abstract}

Keywords: ek-regular homomorphism, preradical, Ş-semimodule, subtractive Ş-subsemimodule.

\section{Introduction:}

Throughout this work, Ş stands for a commutative semiring with identity and a semimodule means a unitary left Ş-semimodule. An Ş-subsemimodule $L$ of an Ş-semimodule $A$ is called subtractive if for all $a, a^{\prime} \in A, a+a^{\prime}, a \in L$ implies $a^{\prime} \in L$ it is clear that 0 and $A$ are subtractive Şsubsemimodules of $A$. An Ş-semimodule $A$ is a subtractive S-semimodule if it has only subtractive subsemimodules (1). The category of Şsemimodules will be denoted by $M_{S}$.

In this work we will introduce the concept of ek-regular homomorphism (which are relevant to our work). A homomorphism of Ş-semimodules $\varphi: A \rightarrow B$ is said to be epimorphism-kernel regular (ek-regular) if $\varphi$ is an epimorphism and k-regular.

In module theory, a preradical $P$ on $R$-Mod (the category of unitary left $R$-modules) is any subfunctor of the identity functor of $R$-Mod (2). The concept of preradical in semimodules was not found in the literatures.

In this paper we study the notion of preradical on some subcategories of the category of semimodules and homomorphism of semimodules. Since some of the known preradicals on modules fail to satisfy the conditions of preradicals, if the category of modules was extended to semimodules, it is necessary to investigate some subcategories of semimodules, like $M_{\text {ek-Ş̧ }} \quad$ (the category of subtractive semimodules with ek-regular homomorphisms) and the category of subtractive semimodules with homomorphisms $M_{C \varsigma \text { ș }}$.

Department of Mathematics, College of Education for Pure Sciences, University of Babylon, Babylon, Iraq.

*Corresponding authors: sarah1993100@gmail.com
That is only we need to prove some properties of homomorphisms on Ş-semimodule.

In addition to section One, there are five more sections. Section Two consists of the preliminaries that we need in our investigation; some of these were found in the literatures, while the results (Lemma 2.10, which are relevant to our work, and other results in the next sections) are part of our investigation. In section Three, three types of preradicals were investigated, Jacobson radical, socle, and second of semimodule. Singular and torsion of semimodule were studied in Section Four . In Section Five the concepts of reject and inject of semimodule were investigated. Finally, in Section Six, preradicals and their properties and types were introduced applying these concepts to the examples of the previous sections.

\section{Preliminaries}

Some definitions and propositions that needed in this work will be introduced.

Definition 2.1(3). Let Ş be a semiring. A left Şsemimodule $A_{S}$ is a commutative monoid $(A,+, 0)$ for which we have a function $S\} \times A \rightarrow A$, defined by $(s, a) \mapsto s a$ such that $\forall s, t \in$ Ş and $a, a^{\prime} \in A$,

1- $s\left(a+a^{\prime}\right)=s a+s a^{\prime}$.

2- $(s+t) a=s a+t a$.

3- $(s t) a=s(t a)$.

4- $0_{S} a=0_{A}=s 0_{A}$.

If $1_{S} a=a$ holds then a left Ş-semimodule $A$ is called unitary.

Definition 2.2(3). Let $L$ be a subset of a left Şsemimodule $A$ then $L$ is called subsemimodule of $A$ if $L$ is closed under addition and scalar multiplication. In this case it is denoted by $L \hookrightarrow A$. 
Definition 2.3(3). An Ş-subsemimodule $L$ of an Şsemimodule $A$ is called subtractive if for all $a, a^{\prime} \in A \quad a, a+a^{\prime} \in L$ implies $a^{\prime} \in L$.

An Ş-semimodule $A$ is a subtractive Şsemimodule if it has only subtractive subsemimodules.

Definition 2.4(3). Let $A$ and $B$ be Ş-semimodules. A homomorphism from $A$ to $B$ is a map $\varphi: A \rightarrow$ $B$ such that

1- $\varphi\left(a+a^{\prime}\right)=\varphi(a)+\varphi\left(a^{\prime}\right)$ and

2- $\varphi(s a)=s \varphi(a) \quad \forall a, a^{\prime} \in A$ and $s \in S$.

For a homomorphism of Ş-semimodules $\varphi: A \rightarrow$ $B$ we define:

$1-\operatorname{ker}(\varphi)=\{a \in A \mid \varphi(a)=0\}$.

2- $\varphi(A)=\{\varphi(a) \mid a \in A)\}$.

3- $\operatorname{Im}(\varphi)=\left\{b \in B \mid b+f(a)=f\left(a^{\prime}\right)\right.$ for some $a$, $\left.a^{\prime} \in A\right\}$.

A homomorphism of Ş-semimodules $\varphi: A \rightarrow B$ is a

1- Monomorphism, if for any Ş-semimodule $L$ and Ş-homomorphisms $\alpha, \beta: L \rightarrow A$ with $\varphi \circ \alpha=$ $\varphi \circ \beta$ we have $\alpha=\beta$.

2- Epimorphism, if for any SS-semimodule $K$ and Ş-homomorphisms $\alpha, \beta: B \rightarrow K$ with $\alpha \circ \varphi=$ $\beta \circ \varphi$ we have $\alpha=\beta$.

3- Isomorphism if $\varphi$ is monomorphism and epimorphism.

4- Image regular ( $i$-regular), if $\varphi(A)=\operatorname{Im}(\varphi)$.

5- Kernel regular (k-regular) if $\varphi(a)=\varphi\left(a^{\prime}\right) \Rightarrow$ $a+k=a^{\prime}+k^{\prime}$ for some, $k, k^{\prime} \in \operatorname{ker}(\varphi)$.

6- Regular if $\varphi$ is $i$-regular and k-regular.

Definition 2.5. A homomorphism of Şsemimodules $\varphi: A \rightarrow B$ is said to be epimorphismkernel regular (ek-regular) if $\varphi$ is an epimorphism and k-regular.

Definition 2.6(4). The (possibly infinite) sequence of Ş-semimodules $\left\{A_{i}\right\}_{i \in I}$

$\ldots A_{i} \stackrel{\varphi_{i}}{\rightarrow} A_{i+1} \stackrel{\varphi_{i+1}}{\longrightarrow} A_{i+2} \stackrel{\varphi_{i+2}}{\longrightarrow} \ldots$ is said to be:

1- Exact, if $\operatorname{Im} \varphi_{i}=\operatorname{ker} \varphi_{i+1} \forall i \in I$.

2- proper exact, if $\varphi_{i}\left(A_{i}\right)=\operatorname{ker} \varphi_{i+1} \forall i \in I$.

Proposition 2.7(3). Let $A$ and $B$ be Ş-semimodules, then a homomorphism of Ş-semimodules $\varphi: A \rightarrow B$ is:

1- Injective if and only if is a monomorphism.

2- Surjective if and only if is an epimorphism and $\varphi(A) \subseteq B$ is subtractive.

Lemma 2.8 (3). Let $A, B$ be Ş-semimodules and $\varphi \in \operatorname{Hom}_{S}(A, B)$, then

1 - $\operatorname{Im}(\varphi)$ is subtractive.

2- $\varphi(A)$ is subtractive if and only if $\varphi(A)=$ $\operatorname{Im}(\varphi)$.

Proposition 2.9 (5). For any k-regular homomorphism $\varphi: A \rightarrow B$ from a subtractive semimodule $A$ to a semimodule $B$ and any subsemimodule $L$ of $A, \varphi^{-1}(\varphi(L))=L+\operatorname{ker} \varphi$.
The following lemma serves to consider the category of subtractive Ş-semimodules and ek-regular Ş- homomorphisms.

Lemma 2.10. Let $A, B$ and $C$ be Ş-semimodules and $\varphi: A \rightarrow B, \alpha: B \rightarrow C$ be ek-regular Şhomomorphisms then $\alpha \varphi: A \rightarrow C$ is also ekregular.

Proof: Let $\alpha(\varphi(a))=\alpha\left(\varphi\left(a^{\prime}\right)\right)$, since $\alpha$ is ekregular, then we have

$\varphi(a)+k=\varphi\left(a^{\prime}\right)+k^{\prime} \quad$ where $k, k^{\prime} \in$ ker $\alpha \subseteq B=\varphi(A)$.

Then, $k, k^{\prime} \in \varphi(A) \Longrightarrow \varphi(a+x)=\varphi\left(a^{\prime}+x^{\prime}\right)$, for some $x, x^{\prime} \in A$.

So, $a+x+t=a^{\prime}+x^{\prime}+t^{\prime} \quad$ for some $t, t^{\prime} \in$ $\operatorname{ker} \varphi$.

Now,since $\operatorname{ker} \varphi \subseteq \operatorname{ker}(\alpha \varphi) \Rightarrow t, t^{\prime} \in$

$\operatorname{ker}(\alpha \varphi)$, also $x, x^{\prime} \in \operatorname{ker}(\alpha \varphi)$. On the other hand, it is clear that $\alpha \varphi$ is epimorphism; therefore, $\alpha \varphi$ is ek-regular.

Note that for any semimodule $A$, the identity $1_{A}$ is an ek-regular homomorphism.

\section{Jacobson Radical, Socle and Second of Semimodule}

In this section we study firstly, the Jacobson radical of semimodule. Secondly, we study the socle radical of semimodule. Also, we proved some properties of it depending on some definitions and results. Finally, the notion of second module and second radical were introduced in (6), and investigating some properties of them. We extend these notions to semimodules. Although, the notion of second semimodule was given in (7), but we gave a different definition analogous to the definition of (6).

\section{Jacobson Radical of Semimodule}

Definition 3.1.1 (8). Let $A$ be an S-semimodule and $L$ be a non-zero S-subsemimodule of $A$. We say that $L$ is a small (superfluous ) Ş-subsemimodule of $A$ if for every Ş-subsemimodule $U$ of $A, L+U=A$ implies $U=A$. Then we shall denote a small Şsubsemimodule $L$ of Ş-semimodule $A$ by $L \preccurlyeq_{s} A$.

It is known that the image of a small submodule under a homomorphism of modules is small. This is not true, in general for semimodules and homomorphisms of semimodules (see Example(1)(8)). In (8) this property was proved under certain condition on homomorphism. The following lemma uses a weaker condition to prove the same property.

Lemma 3.1.2 Let $A, B$ be subtractive Şsemimodules, $L \preccurlyeq_{s} A$ and let $\varphi \in \operatorname{Hom}_{\mathrm{S}}(A, B)$, if $\varphi$ is ek-regular. Then $\varphi(L) \preccurlyeq_{S} B$.

Proof: Assume that $\varphi(L)+U=B$, where $U \hookrightarrow B$.

Now, Let $a \in A \Longrightarrow \varphi(a)=\varphi(l)+u$, for some $l \in L \wedge u \in U$. 
Since $\varphi(A)$ is subtractive, it follows $u \in \varphi(A)$ and $u=\varphi(x)$ for some $\in A\left(x \in \varphi^{-1}(U)\right)$.

Then $\varphi(a)=\varphi(l+x)$. Since $\varphi$ is ek-regular, we obtain

$a+k=l+x+k^{\prime}$, where, $k^{\prime} \in \operatorname{Ker} \varphi \subseteq$ $\varphi^{-1}(U) \Rightarrow \quad a \in L+\varphi^{-1}(U)$. Thus $A=L+$ $\varphi^{-1}(U)$.

Since $L \preccurlyeq_{S} A$, it follows $\varphi^{-1}(U)=A$. Thus $U=B$ ( $\varphi$ is an epimorphism). Hence $\varphi(L) \preccurlyeq_{s} B$.

Recall that a maximal S-subsemimodule of a semimodule $A$ is a proper subsemimodule of $A$ that is not contained properly in any other proper subsemimodule of $A$.

Definition 3.1.3(8). Let $A$ be an Ş-semimodule, the Jacobson radical of $A$ is denoted by $J(A)$ and defined as

$$
=\bigcap_{K \subseteq A}^{J(A)}\{K
$$

: $K$ is a maximal subsemimodule of $A\}$.

Proposition 3.1.4(8). Let $A$ be an Ş-semimodule, then

$$
\sum_{L \preccurlyeq_{s} A} L
$$$$
=\bigcap_{K \subseteq A}\{K: \text { is a maximal subsemimodule of } A\} .
$$

Proposition 3.1.5. Let $A$ be an Ş-semimodule then:

1- $J(A) \hookrightarrow A$.

2- Let $A$ and $B$ be subtractive Ş-semimodules and $\varphi \in \operatorname{Hom}_{\widehat{S}}(A, B)$ be ek-regular Şhomomorphism, then $\varphi(J(A)) \subseteq J(B)$.

Proof: (1) it is obvious by Definition(3.1.3).

(2) by Proposition(3.1.4) $J(A)=\sum_{L \preccurlyeq_{S} A} L$.

Then $\varphi(J(A))=\varphi\left(\sum_{L \preccurlyeq_{S} A} L\right)=\sum_{L \preccurlyeq_{S} A} \varphi(L)$,

Since $\varphi$ is ek-regular, so by the Lemma(3.1.2).

$\varphi(J(A))=\varphi\left(\sum_{L \preccurlyeq_{S} A} L\right)=\sum_{\varphi(L) \preccurlyeq_{S} B} \varphi(L) \subseteq$ $\sum_{K \preccurlyeq_{s} B} K=J(B)$.

Note that the previous result (2), was proved in (8), with condition on $\varphi$, k-quasiregular, but we proved the result with the weaker condition $\mathrm{k}$ regular.

Lemma 3.1.6. Let $A$ be an Ş-semimodule, $\tilde{U}$ is maximal in $A / C$ if and only if $U=v^{-1}(\tilde{U})$ is maximal in $A$.

Proof: $\Rightarrow$ Assume that $v: A \rightarrow A / C$ be an SShomomorphism and. $\tilde{U}$ is maximal in $A / C$, then $U=v^{-1}(\tilde{\mathrm{U}}) \leftrightarrow \tilde{\mathrm{U}}=U / C$. To prove $U$ is maximal in $A$.

Now, let $U \subseteq V \subseteq A \Rightarrow \tilde{\mathrm{U}} \subseteq V / C \subseteq A / C$ for some subsemimodule $V$ of $A$ containing $C$.

Then $V / C=\tilde{\mathrm{U}}$ or $V / C=A / C$ (since $\tilde{\mathrm{U}}$ is maximal ), That is $V=U$ or $V=A$.

Hence $U$ is a maximal in $A$.
$\Leftarrow$ Assume that $U=v^{-1}(\tilde{\mathrm{U}})$ is maximal in $A$ and let $\tilde{\mathrm{U}} \subseteq \tilde{V} \subseteq A / C$, but $\tilde{V}=V / C$ for some subsemimodule $V$ of $A$ containing $C$.

So, $U / C \subseteq V / C \subseteq A / C$, which implies $U \subseteq V \subseteq A$. Since $U$ is maximal in $A$,

Thus $V=U$ or $V=A$, that is $\tilde{\mathrm{U}}=\tilde{V} \quad$ or $\quad \tilde{V}=$ $A / C$.

Hence $\tilde{U}$ is a maximal in $A / C$.

Proposition 3.1.7. Let $A$ be an Ş-semimodule and $J$ is a Jacobson radical of $A$ then $J(A / J(A))=0$.

Proof: Let

$J(A / J(A))=$

$\bigcap\{\tilde{U} \mid \tilde{U}$ is maximal subsemimodule in $A / J(A)\}$

and $J(A)=C$.

Since U is maximal subsemimodule in $A / J(A) \Rightarrow$ $\tilde{\mathrm{U}}=U / C$, (where $U$ is a maximal subsemimodule in $A$ and containing $C$ by Lemma (3.1.6)).

Then

$J(A / J(A))=$

$\bigcap\{\tilde{U} \mid \tilde{U}$ is maximal subsemimodule of $A / J(A)\}$

$$
\begin{aligned}
& =\left\{\frac{U}{J(A)} \mid U \text { is maximal subsemimodule of } A\right. \text { and } \\
& \quad \text { containing } J(A)\} \\
& =\cap U / J(A) \\
& =J(A) / J(A)=0 .
\end{aligned}
$$

\section{Socle Radical of Semimodule}

Recall that a non-zero S-semimodule $A$ is called simple if it has no proper non-zero Şsubsemimodule.

Definition 3.2.1(5). Let $A$ be an Ş-semimodule. The Socle radical of $A$ is denoted by $\operatorname{Soc}(A)$ and defined as

$\operatorname{Soc}(A)=\sum\{L: L$ is a simple Ş-subsemimodule of $A\}$.

Lemma 3.2.2. Let $A$ and $B$ be subtractive Şsemimodules and $\varphi \in \operatorname{Hom}_{\widehat{S}}(A, B)$ be ek-regular Ş-homomorphism, if $L$ is a simple Ş-subsemimodule of $A$ then $\varphi(L)$ is simple Ş-subsemimodule of $B$.

Proof: Let $L$ be simple Ş-subsemimodule of $A$.

If $0 \neq U \hookrightarrow \varphi(L) \Rightarrow$ either $\varphi^{-1}(U)=0 \Rightarrow U=0$ (which is a contradiction).

Or $\varphi^{-1}(U)=L \Rightarrow U=\varphi(L) \quad$ (since $\varphi$ is an epimorphism).

Then $0 \neq \varphi^{-1}(U) \hookrightarrow \varphi^{-1}(\varphi(L))$.

Now, since $\varphi$ is k-regular, so by Proposition (2.9) we have

$\varphi^{-1}(U) \hookrightarrow L+\operatorname{Ker} \varphi \Rightarrow \varphi^{-1}(U) \hookrightarrow L \quad$ (which is a contradiction).

Proposition 3.2.3. Let $A$ be an Ş-semimodule then:

$1-\operatorname{Soc}(A) \hookrightarrow A$.

2- Let $A$ and $B$ be subtractive Ş-semimodules and $\varphi \in \operatorname{Hom}_{\widehat{S}}(A, B)$, if $\varphi$ is ek-regular then $\varphi(\operatorname{Soc}(A)) \subseteq \operatorname{Soc}(B)$.

Proof: (1) it is clear by Definition(3.2.1). 
(2) by $\operatorname{Definition(3.2.1),~} \operatorname{Soc}(A)=\sum\{L$ : $L$ is a simple $S$-subsemimodule of $A\}$.

Then, $\varphi(\operatorname{Soc}(A))=\varphi\left(\sum L\right)=\sum \varphi(L)$

( $L$ is a simple SS-subsemimodule of $A$ ).

Since $\varphi$ is ek-regular, so by Lemma (3.2.2), we have

$\varphi(\operatorname{Soc}(A))=\sum\{\varphi(L): L \quad$ is a simple $\quad$ subsemimodule of $A\}$

$$
=\sum\{\varphi(L): \varphi(\mathrm{L}) \text { is a simple Ş- }
$$

subsemimodule of $B\}$

$\subseteq \sum\{K: K$ is a simple $S$-subsemimodule

of $B\}=\operatorname{Soc}(B)$.

\section{Second Radical of Semimodule:}

Recall that a left annihilator of $x$ in $S S$ is defined by $\operatorname{ann}_{S}(x)=\{s \in S \mid s x=0\}$, it is clear that $a n n_{S}(x)$ is a left ideal of Ş. Also if $L$ is a subsemimodule of $A$, then $a n n_{S}(L)=$ $\{s \in S \mid s x=0 \forall x \in L\}$.

Definition 3.3.1 An Ş-semimodule $A$ is called a second semimodule where $A \neq 0$ and $\operatorname{ann}_{\widehat{S}}(A)=$ $\operatorname{ann}_{\widehat{S}}(A / L) \forall L \subseteq A$. Any subsemimodule of $A$ is said to be second if it is a second semimodule.

Definition 3.3.2. Let $A$ be an S-semimodule. The Second radical of $A$ is denoted by $\sec (A)$ and defined as

$\operatorname{Sec}(A)=\sum\{L: L$ is a second Ş-subsemimodule of $A\}$.

Lemma 3.3.3. Let $A$ and $B$ be subtractive Şsemimodules and $\varphi \in \operatorname{Hom}_{S}(A, B)$ be k-regular, if $L$ is a second S-subsemimodule of $A$ then $\varphi(L)$ is second Ş-subsemimodule of $B$.

Proof: Let $L$ be second Ş-subsemimodule of $A \Rightarrow$ $\operatorname{ann}_{\text {Ş}}(L) \supseteq \operatorname{ann}_{\text {Ş}}(L / K), \quad \forall K \hookrightarrow L \wedge K \neq L$.

And let $K^{\prime} \hookrightarrow \varphi(L) \wedge s \varphi(L) \subseteq K^{\prime} \quad \forall s \in S$.

Now $s \varphi(L) \subseteq K^{\prime} \Rightarrow \varphi(s L) \subseteq K^{\prime} \quad \forall s \in S$

So, $\quad \varphi^{-1}(\varphi(s L)) \subseteq \varphi^{-1}\left(K^{\prime}\right) \Rightarrow s L+k \operatorname{er} \varphi \subseteq$ $\varphi^{-1}\left(K^{\prime}\right) \quad$ (by Proposition(2.9)).

We have $s L \subseteq K \Longrightarrow s L=0 \quad \forall s \in$ S.

Thus $s L \subseteq \varphi^{-1}\left(K^{\prime}\right) \Rightarrow s L=0 \Rightarrow s \varphi(L)=0$.

Hence $\varphi(L)$ is second Ş-subsemimodule of $B$.

$\square$

Proposition 3.3.4. Let $A$ be an Ş-semimodule then:

$1-\operatorname{Sec}(A) \hookrightarrow A$.

2- Let $A$ and $B$ be subtractive S-semimodules and $\varphi \in \operatorname{Hom}_{\mathrm{S}}(A, B)$, if $\varphi$ is k-regular then $\varphi(\operatorname{Sec}(A)) \subseteq \operatorname{Sec}(B)$.

Proof: (1) it is clear by Definition(3.3.2).

(2) by $\operatorname{Definition}(3.3 .2), \quad \operatorname{Sec}(A)=\sum\{L$ : $L$ is a second SS-subsemimodule of $A\}$

Then, $\varphi(\operatorname{Sec}(A))=\varphi\left(\sum L\right)=\sum \varphi(L)$ ( is a second Ş-subsemimodule of $A$ )

Since $\varphi$ is k-regular, so by Lemma (3.3.3), we have $\varphi(\operatorname{Sec}(A))=\sum\{\varphi(L): L$ is a second Ş-

subsemimodule of $A\}$
$=\sum\{\varphi(L): \varphi(\mathrm{L})$ is a second Ş-

subsemimodule of $B\}$

$\subseteq \quad \sum\{K:$ is a second Ş-subsemimodule of $B\}=\operatorname{Sec}(B)$.

\section{Singular and Torsion of Semimodule}

In this section, we study two concepts singular subsemimodule and torsion subsemimodule and some characterization of them.

\section{Singular of Semimodule}

Definition 4.1.1(9): An ideal $I$ of a semiring $S$ is said to be an essential ideal of Ş if for every ideal $K$ of $S, I \cap K=0$ implies $K=0$. Then we shall denote an essential ideal $I$ of a semiring Şsemimodule by $I \unrhd_{e} S$ S .

Definition 4.1.2(9): Let $A$ be an Ş-semimodule, a singular subsemimodule of $A$ is denoted by $Z(A)$ and defined as $Z(A)=\left\{a \in A \mid a n n_{S}(a) \unrhd_{e} S\right\}$.We say that $A$ is singular if $Z(A)=A$ and nonsingular if $\mathcal{Z}(A)=0$.

Proposition 4.1.3. Let $A$ be an Ş-semimodule and $L$ be SS-subsemimodule of $A$ then:

$1-z(A) \hookrightarrow A$.

2- $\quad Z(L)=L \cap Z(A)$.

3- $\quad Z(Z(A))=Z(A)$.

4- Let $A$ and $B$ be S-semimodules, if $\varphi \in$ $H_{S o m}(A, B)$ then $\varphi(Z(A)) \subseteq Z(B)$.

Proof: (1),(2) and (3) they are clear by Definition(4.1.2).

(4)

$a \in Z(A) \Longrightarrow \operatorname{ann}_{\widehat{S}}(\varphi(a)) \supseteq \operatorname{ann}_{\widehat{S}}(a) \unrhd_{e} S \Longrightarrow$ $\operatorname{ann}_{S}(\varphi(a)) \unrhd_{e}$ Ş. Thus $\varphi(a) \in Z(B)$.

Hence $\varphi(Z(A)) \subseteq Z(B)$.

\section{Torsion of Semimodule}

Definition 4.2.1(10): Let $A$ be an S-semimodule. An element $a \in A$ is said to be torsion element if there exists $0 \neq s \in S$ such that $s a=0$. A torsion subsemimodule of $A$ is denoted by $\mathcal{T}(A)$ and define as $\mathcal{T}(A)=\left\{a \in A \mid \begin{array}{c}s a=0 \\ \text { for some } 0 \neq s \in S\end{array}\right\}$.We say that $A$ is torsion if $\mathcal{T}(A)=A$ and torsion free if $\mathcal{T}(A)=0$.

In order to have $\mathcal{T}(A)$ a subsemimodule of $A$. We need to convert Definition (4.2.1) to the following:

Definition 4.2.2. Let $A$ be a semimodule over a commutative semiring S. An element $a \in A$ is said to be torsion element if $s a=0$, for some nonzero divisor $s \in$ Ş. then

$\mathcal{T}(A)=$

$\{a \in A \mid s a=0$ for some nonzero divisor $s \in S\}$.

Proposition 4.2.3. Let $A$ be an Ş-semimodule and $L$ be Ş-subsemimodule of $A$ then:

$1-\mathcal{T}(A) \hookrightarrow A$.

2- $\mathcal{T}(L)=L \cap \mathcal{T}(A)$. 
3- $\mathcal{T}(\mathcal{T}(A))=\mathcal{T}(A)$.

4- $\mathcal{T}(A / \mathcal{T}(A))=0$.

5- Let $A$ and $B$ be S-semimodules, if $\varphi \in$ $\operatorname{Hom}_{S}(A, B)$ then $\varphi(\mathcal{T}(A)) \subseteq \mathcal{T}(B)$.

Proof: (1),(2) and (3) they are clear by Definition(4.2.1).

(4) Let $0 \neq a+\mathcal{T}(A) \in A / \mathcal{T}(A) \Rightarrow a \notin \mathcal{T}(A)$

Now, if $s(a+\mathcal{T}(A))=0$, then $s a+\mathcal{T}(A)=0$, so $s a \in \mathcal{T}(A)$ for some nonzero divisor $s \in S$.

Hence $t(s a)=0$, for some nonzero divisor $t \in S$ S implies $(t s) a=0, \quad$ that is, $\quad a \in \mathcal{T}(A) \quad$ a contradiction.

Therefore $a+\mathcal{T}(A)$ is not torsion. Thus $\mathcal{T}(A$ / $\mathcal{T}(A))=0$.
(5)
By
Definition
(4.2.1),

$\mathcal{T}(A)=$

$\{a \in A \mid s a=0$ for some nonzero divisor $s \in$ S $\}$.

Now,

$a \in \mathcal{T}(A) \Rightarrow s a=$

0 for some nonzero divisor $s \in$ S.

$\Longrightarrow \varphi(s a)=0$, for some nonzero divisor $s \in$ SS.

$\Rightarrow s \varphi(a)=0$ for some nonzero divisor $s \in S$.

Thus $\varphi(a) \in \mathcal{T}(B)$.

Hence $\varphi(\mathcal{T}(A)) \subseteq \mathcal{T}(B)$.

\section{Reject and Inject of Semimodule}

In the following we need to define subcategories of the category of Ş-semimodule and Ş-homomorphism.

1- The category of subtractive Ş-semimodules and Ş-homomorphism, which will be denoted by $M_{S}$.

2- The category of subtractive S-semimodules and ek-regular (see Lemma 2.10), which will be denoted by $M_{\text {ek-ș. }}$.

Definition 5.1 (1)(p.54). Let $\Delta$ be a class of semimodules. The reject of an Ş-semimodule $A$ denoted by $\mathrm{R}_{\Delta}(A)$ is defined by $\mathrm{R}_{\Delta}(A)=\mathrm{n}$ $\operatorname{ker} f\left\{f \in H_{\text {om }}(A, B), B \in \Delta\right\}$.

Proposition 5.2 (1)(p.58). Let $\Delta$ be a class of all Şsemimodules. If $A, B \in \Delta$ and $f \in \operatorname{Hom}_{S}(A, B)$, then $f\left(\mathrm{R}_{\Delta}(A)\right) \subseteq \mathrm{R}_{\Delta}(B)$.

The following operator has an analogous in modules with abbreviation "Trace", we will define it for semimodules using another name and symbol convenient to Definition(5.1).

Definition 5.3. Let $\Delta$ be a class of semimodules and $A$ an SS-semimodule. The inject of $A$ denoted by $b^{\Delta}(A)$ and defined as $b^{\Delta}(A)=\sum \operatorname{Im} f\{f \in$ $\left.H_{\text {orm }}(B, A), B \in \Delta\right\}$.

Proposition 5.4. Let $\Delta$ be a class of all subtractive Ş -semimodules. If $A, B \in \Delta$ and $f \in \operatorname{Hom}_{\widehat{S}}(A, B)$, then $f\left(b^{\Delta}(A)\right) \subseteq b^{\Delta}(B)$.

Proof: Suppose $x \in b^{\Delta}(A)$ and $h \in \operatorname{Hom}_{\mathrm{S}}(L, B), L \in$ $\Delta$ then $h f \in \operatorname{Hom}_{\mathrm{S}}(L, A)$
$\Rightarrow f(x) \in I m h$. (Note that in $M_{c \S} \operatorname{Imh}=h(L)$, see 2.7(2))

Hence $f\left(b^{\Delta}(A)\right) \subseteq b^{\Delta}(B)$.

\section{Preradical on Semimodules}

This section consists of definitions and examples about preradicals on semimodules analogous to the cases in modules (see (2)), also some results, to solve the problems arises through the extension of the category of modules to that of semimodules, are proved in the category of semimodules or some subcategories of it.

Definition 6.1. A functor P: $M_{S} \rightarrow M_{S}$ is called preradical on $M_{S}$ if the following two conditions are satisfied:

1- $\mathrm{P}(A) \hookrightarrow A, \forall A \in M_{\mathrm{S}}$.

2- $\forall \varphi \in \operatorname{Hom}_{\mathrm{S}}(A, B) \varphi(\mathrm{P}(A)) \subseteq \mathrm{P}(B)$.

In this definition (and what follow) the category $M_{S}$, can be replaced by $M_{c S}$ (The subcategory of subtractive Ş-semimodules and Şhomomorphism) or by $M_{\text {ek-S }}$ (the subcategory of subtractive Ş-semimodules and ek-regular homomorphisms).

\section{Examples 6.2.}

6.2.1 By Propositions (3.1.5), (3. 2.3) and (3.3.4) the functors $J, \delta o c$ and $\delta e c$ are preradicals on the category $M_{\text {et-ş. }}$.

6.2.2 By Propositions $(4.1 .3(1,4))$ and $(4.2 .3(1,5))$ the functors $\mathcal{Z}$ and $\mathcal{T}$ are preradicals on the category $M_{S}$.

6.2.3 By Proposition (5.2) a functor $\mathrm{R}_{\Delta}$ is preradical on the category $M_{S}$ and by Proposition (5.7) the functor $b^{\Delta}$ is preradical on the category $M_{C S ̧}$.

Definitions 6.3. $\forall A \in M_{S}$, a $\quad$ Preradical $P$ is called,

1- Radical if $\mathrm{P}(A / \mathrm{P}(A))=0$.

2- Idempotent if $\mathrm{P}(\mathrm{P}(A))=\mathrm{P}(A)$.

3- Hereditary if $\mathrm{P}(L)=L \cap \mathrm{P}(A)$, with $L \hookrightarrow A$.

Examples 6.4.

6.4.1 The preradicals $J$ and $\mathcal{T}$ are radicals by Proposition ((3.1.7),(4.2.2)(4))) .

6.4.2 The preradical $S o c$ is hereditary and idempotent (by (5), Corollary 3.11), and also the preradicals $Z, \mathcal{T}$ are hereditary and idempotent by Propositions (4.1.3(2,3), (4.2.3(2,3))).

Note 6.5. Let $\mathrm{P}$ be a preradical $\forall A \in M_{\mathrm{S}}$. If $\left\{\mathrm{P}_{i}: i \in I\right\}$ is a collection of preradicals then

1- $\bigcap_{i \in I} \mathrm{P}_{i}, \quad$ is defined as $\left(\bigcap_{i \in I} \mathrm{P}_{i}\right)(A)=$ $\bigcap_{i \in I} P_{i}(A)$, is preradical.

2- $\sum_{i \in I} \mathrm{P}_{i}, \quad$ is defined as $\left(\sum_{i \in I} \mathrm{P}_{i}\right)(A)=$ $\sum_{i \in I} \mathrm{P}_{i}(A)$, is preradical.

3- Let $\mathrm{P}, \mathrm{t}$ be two preradicals, then $\mathrm{P} \leq \mathrm{t}$, if $\mathrm{P}(A)) \subseteq \mathrm{\textrm {L }}(A), \forall A \in M_{\mathrm{s}}$.

Definition 6.6. Let $\mathcal{M}$ be a class of semimodules, $\mathcal{M}$ is said to be, 
1- Hereditary if $\mathcal{M}$ is closed under isomorphisms and subsemimodules.

2- Cohereditary if $\mathcal{M}$ is closed under epimorphic images.

Definition 6.7. Let $\mathrm{P}$ be a preradical, an Şsemimodule $A \in M_{\mathrm{S}}$ is said to be ,

1- P-torsion if $\mathrm{P}(A)=A$. A class of all P-torsion semimodules is denoted by $\mathrm{T}_{\mathrm{P}}$.

2- P-torsionfree if $\mathrm{P}(A)=0$. A class of all $\mathrm{P}$ torsionfree semimodules is denoted by $\mathrm{F}_{\mathrm{P}}$.

Proposition 6.8. Let $A \in M_{S}$ and $\mathrm{P}$ be a preradical , $\forall K \hookrightarrow A$, then:

1- $\mathrm{P}(K) \subseteq K \cap \mathrm{P}(A)$.

2- $(\mathrm{P}(A)+K) / K \subseteq \mathrm{P}(A / K)$.

3- If $\mathrm{P}(A / K)=0$ then $\mathrm{P}(A) \subseteq K$.

4- If $\mathrm{P}(K)=K$ then $K \subseteq \mathrm{P}(A)$.

Proof: (1) and (2) by definition of preradical. (3) by (2) and (4) by (1).

Proposition 6.9. Let $\mathrm{P}$ be a preradical. If $\left\{A_{i}: i \in\right.$ $I\}$ is a collection of S-semimodules then:

1- $\mathrm{P}\left(\bigoplus_{i \in I} A_{i}\right)=\bigoplus_{i \in I} \mathrm{P}\left(A_{i}\right)$.

2- $\mathrm{P}\left(\prod_{i \in I} A_{i}\right) \subseteq \prod_{i \in I} \mathrm{P}\left(A_{i}\right)$.

Proof: Since $\mathrm{P}$ is a subfunctor of the identity functor.

Proposition 6.10. Let $P$ be a preradical, then:

1- $T_{\mathrm{P}}$ is a cohereditary class closed under arbitrary direct sums.

2- $F_{\mathrm{P}}$ is a hereditary class closed under arbitrary direct products.

Proof: (1). Let $A \in \mathrm{T}_{\mathrm{P}} \Rightarrow \mathrm{P}(A)=A, \varphi: A \rightarrow B$ be an epimorphism.

Then $\quad \varphi(\mathrm{P}(A)) \subseteq \mathrm{P}(B) \Rightarrow \varphi(A) \subseteq \mathrm{P}(B)$ (since $\mathrm{P}(A)=A$ )

$\Longrightarrow B \subseteq \mathrm{P}(B)$

(since $\varphi$ is onto)

....(i).

But $\mathrm{P}(B) \subseteq B \quad$ (by definition)

So, from (i) and (ii) we have $\mathrm{P}(B)=B \Rightarrow B \in \mathrm{T}_{\mathrm{P}}$. Now, let $\left\{A_{i}: i \in I\right\} \subseteq \mathrm{T}_{\mathrm{P}} \Rightarrow \mathrm{P}\left(A_{i}\right)=A_{i} \forall i \in I$.

Then $\mathrm{P}\left(\bigoplus_{i \in I} A_{i}\right)=\bigoplus_{i \in I} \mathrm{P}\left(A_{i}\right)=\bigoplus_{i \in I} A_{i} \quad \Rightarrow$ $\bigoplus_{i \in I} A_{i} \in \mathrm{T}_{\mathrm{P}}$.

Hence $T_{P}$ is a cohereditary class closed under arbitrary direct sums.

(2). Let $A \in \mathrm{F}_{\mathrm{P}} \Rightarrow \mathrm{P}(A)=0$.

So, if $K \subseteq A \Rightarrow \mathrm{P}(K) \subseteq K \cap \mathrm{P}(A) \quad \Rightarrow \mathrm{P}(K) \subseteq$ $K \cap 0=0 \Rightarrow \mathrm{P}(K)=0$.

Thus $K \in \mathrm{F}_{\mathrm{P}}$.

Now, let $\varphi: A \rightarrow K$ be an isomorphism and $A \in \mathrm{F}_{\mathrm{P}}$. Then $\varphi^{-1}(\mathrm{P}(K)) \subseteq \mathrm{P}(A)=0 \Rightarrow \mathrm{P}(K) \subseteq$ $\operatorname{ker} \varphi^{-1}=0$, So $K \in \mathrm{F}_{\mathrm{P}}$.

Now, let $\left\{A_{i}: i \in I\right\} \subseteq \mathrm{F}_{\mathrm{P}} \Rightarrow \mathrm{P}\left(A_{i}\right)=0 \forall i \in I$. Since $\mathrm{P}\left(\prod_{i \in I} A_{i}\right) \subseteq \prod_{i \in I} \mathrm{P}\left(A_{i}\right)=0 \Rightarrow \prod_{i \in I} A_{i} \in$ $\mathrm{F}_{\mathrm{P}}$

Hence $F_{\mathrm{P}}$ is a hereditary class closed under arbitrary direct products.

Proposition 6.11. Where $\mathrm{P}$ is a preradical, then $1-\operatorname{Hom}_{S}(T, F)=0, \forall T \in \mathrm{T}_{\mathrm{P}}$ and $F \in \mathrm{F}_{\mathrm{P}}$.
2- $\mathrm{T}_{\mathrm{P}} \cap \mathrm{F}_{\mathrm{P}}=0$.

Proof: (1). Let $T \in \mathrm{T}_{\mathrm{P}}, F \in \mathrm{F}_{\mathrm{P}}$ and $\varphi: T \rightarrow F$, then $\varphi(T)=\varphi(\mathrm{P}(T)) \subseteq \mathrm{P}(F)=0$. So $\varphi=0$.

(2). Let $N \in \mathrm{T}_{\mathrm{P}} \cap \mathrm{F}_{\mathrm{P}}$, then $N=\mathrm{P}(N)=0$.

Proposition 6.12. Let $P$ be a radical, then:

1- $T \in \mathrm{T}_{\mathrm{P}}$ if and only if $\operatorname{Hom}_{\mathrm{S}}(T, F)=0, \forall F \in$ $\mathrm{F}_{\mathrm{P}}$.

2- $\mathrm{T}_{\mathrm{P}}$ is closed under extensions.

Proof: (1). By Proposition (6.11), it is enough to prove the sufficiency.

Let $H_{\mathrm{Som}}(T, F)=0, \forall F \in \mathrm{F}_{\mathrm{P}}$.

Since $T / \mathrm{P}(T) \in \mathrm{F}_{\mathrm{P}}(\mathrm{P}$ is a radical), then the natural map $T \rightarrow T / \mathrm{P}(T)$ is zero, hence $\mathrm{P}(T)=T \in \mathrm{T}_{\mathrm{P}}$.

2. Follows from 1.

Proposition 6.13. Let $\mathrm{P}$ be an idempotent Preradical, then:

1- $F \in \mathrm{F}_{\mathrm{P}}$ if and only if $\operatorname{Hom}_{\mathrm{S}}(T, F)=0, \forall T \in$ $T_{P}$.

2- $F_{\mathrm{P}}$ is closed under extensions.

Proof: (1). By Proposition (6.11), it is enough to prove the sufficiency.

Now, let $\operatorname{Hom}_{S}(T, F)=0, \forall T \in \mathrm{T}_{\mathrm{P}}$.

Since $\mathrm{P}(F) \in \mathrm{T}_{\mathrm{P}}(\mathrm{P}$ is an idempotent $)$, then the inclusion map $\mathrm{P}(F) \rightarrow F$ is a zero map, hence $\mathrm{P}(F)=0$.

That is $F \in \mathrm{F}_{\mathrm{P}}$.

(2). Follows from 1.

Proposition 6.14. Let $\left\{A_{i}: i \in I\right\}$ be a collection of S-semimodules then:

1- If $A_{i} \in \mathrm{T}_{\mathrm{P}} \forall i \in I$, then $\sum_{i \in I} A_{i} \in \mathrm{T}_{\mathrm{P}}$.

2- If $A / A_{i} \in \mathrm{F}_{\mathrm{P}} \forall i \in I$, then $A / \bigcap_{i \in I} A_{i} \in \mathrm{F}_{\mathrm{P}}$.

Proof: (1) since $A_{i} \in \mathrm{T}_{\mathrm{P}} \Rightarrow \mathrm{P}\left(A_{i}\right)=A_{i}$

Then $\mathrm{P}\left(\sum_{i \in I} A_{i}\right)=\sum_{i \in I} \mathrm{P}\left(A_{i}\right)=\sum_{i \in I} A_{i}$.

Hence $\sum_{i \in I} A_{i} \in \mathrm{T}_{\mathrm{P}}$.

(2) since $A / A_{i} \in \mathrm{F}_{\mathrm{P}} \Rightarrow \mathrm{P}\left(A / A_{i}\right)=0$.

For each $j \in I$, let $\varphi_{j}: A / \cap A_{i} \rightarrow A / A_{j}$ be a natural epimorphism $\left(a+\cap A_{i} \mapsto a+A_{j}\right)$, then

$\varphi_{j}\left(\mathrm{P}\left(A / \cap A_{i}\right)\right) \subseteq \mathrm{P}\left(A / A_{j}\right)=0$, so $\mathrm{P}\left(A / \cap A_{i}\right) \subseteq$ $\operatorname{ker} \varphi_{j}=A_{j} / \cap A_{i}$ for each $j$, hence

$\mathrm{P}\left(A / \cap A_{i}\right) \subseteq \cap$ ker $f_{j}=\cap A_{j} / \cap A_{i}=0$. Hence $A / \cap$ $A_{i} \in \mathrm{F}_{\mathrm{P}}$.

Recall that a functor $\mathcal{F}$ is said to be left exact, if from a given exact sequence

$0 \rightarrow A \stackrel{\varphi}{\rightarrow} M \stackrel{\alpha}{\rightarrow} \mathrm{W} \rightarrow 0$ we get a left exact sequence $0 \longrightarrow \mathcal{F}(A) \stackrel{\mathcal{F}(\varphi)}{\longrightarrow} \mathcal{F}(M) \stackrel{\mathcal{F}(\alpha)}{\longrightarrow} \mathcal{F}(\mathrm{W})$.

Proposition 6.15. Let $\mathrm{P}$ be a preradical on $M_{c S}$, then the following are equivalent:

1- $\mathrm{P}$ is left exact as a functor

2- $\mathrm{P}$ is hereditary

3- $\mathrm{P}$ is idempotent and $\mathrm{T}_{\mathrm{P}}$ is hereditary.

Proof: (1) $\Rightarrow(2)$ let $N$ be a subsemimodule of $M$ then by (1), the sequence

$0 \rightarrow \mathrm{P}(N) \rightarrow \mathrm{P}(M) \rightarrow \mathrm{P}(M / N)$ is exact, so $\mathrm{P}(N)=N \cap \mathrm{P}(M)$. 
$(2) \Rightarrow(3)$ let $\mathrm{P}$ be hereditary, then we have $\mathrm{P}(\mathrm{P}(M))=\mathrm{P}(M) \cap \mathrm{P}(M)=\mathrm{P}(M)$. So $\mathrm{P}$ is an idempotent.

Now, if $K \subseteq M$ and $\quad M \in \mathrm{T}_{\mathrm{P}} \Rightarrow \mathrm{P}(K)=K \cap$ $\mathrm{P}(M)=K \cap M=K$.

Hence $K \in \mathrm{T}_{\mathrm{P}}$ and $\mathrm{T}_{\mathrm{P}}$ is hereditary.

(3) $\Rightarrow$ (1) let $0 \rightarrow N \stackrel{\varphi}{\rightarrow} M \stackrel{\alpha}{\rightarrow} \mathrm{W} \rightarrow 0$ be an exact sequence.

Then the sequence $0 \longrightarrow \mathrm{P}(N) \stackrel{\mathrm{P}(\varphi)}{\longrightarrow} \mathrm{P}(M) \stackrel{\mathrm{P}(\alpha)}{\longrightarrow} \mathrm{P}(\mathrm{W})$, where $\mathrm{P}(\varphi)$ and $\mathrm{P}(\alpha)$ are restrictions of $\varphi$ and $\alpha$. The exactness in $\mathrm{P}(N)$ is clear also the inclusion $\operatorname{ImP}(\varphi) \subseteq \operatorname{kerP}(\alpha)$.

Since $\quad \mathrm{P}(\operatorname{Im} \varphi \cap \mathrm{P}(M))=(\operatorname{Im} \varphi \cap \mathrm{P}(M)) \cap$ $\mathrm{P}(\mathrm{P}(M))=\operatorname{Im} \varphi \cap \mathrm{P}(M) \quad$... (i).

And $\quad \mathrm{P}(\operatorname{Im} \varphi \cap \mathrm{P}(M)) \subseteq \mathrm{P}(\operatorname{Im} \varphi)=\operatorname{Im} \mathrm{P}(\varphi)$ ...(ii).

From (i) and (ii) $\Rightarrow \operatorname{Im} \varphi \cap \mathrm{P}(M) \subseteq \operatorname{Im} \mathrm{P}(\varphi)$ ...(iii).

Now let $a \in \operatorname{ker} \mathrm{P}(\alpha)$ be an arbitrary element $\Rightarrow$ $a \in \mathrm{P}(M) \wedge \alpha(a)=0 \Rightarrow a \in \mathrm{P}(M) \wedge a \in \operatorname{Im} \varphi$.

Thus $a \in \operatorname{Im} \varphi \cap \mathrm{P}(M)$.

So by (i) $a \in \operatorname{Im} \mathrm{P}(\varphi) \Rightarrow \operatorname{ker} \mathrm{P}(\alpha) \subseteq \operatorname{ker} \mathrm{P}(\varphi)$ as required.

Theorem 6.16. If $\mathrm{P}$ is a Preradical on $M_{c \varsigma}$, then the following are equivalent

1- $P$ is an idempotent radical.

2- $\forall A \in M_{S}\left(M_{C S}\right)$ there exists a uniquely determined (up to isomorphism ) proper exact sequence(exact sequence) $\quad 0 \rightarrow T \rightarrow A \rightarrow F \rightarrow$ $0, F \in \mathrm{F}_{\mathrm{P}}, T \in \mathrm{T}_{\mathrm{P}}$.

3- $\forall A \in M_{c S}$ there exists an exact sequence(proper exact sequence) $0 \rightarrow T \rightarrow A \rightarrow F \rightarrow 0, F \in \mathrm{F}_{\mathrm{P}}$, $T \in \mathrm{T}_{\mathrm{p}}$.

4- $\mathrm{P}$ is an idempotent radical and $\mathrm{T}_{\mathrm{P}}, \mathrm{F}_{\mathrm{P}}$ are closed under extensions.

Proof:(1) $\Rightarrow(2) . \quad$ Let $\quad 0 \rightarrow \mathrm{P}(A) \rightarrow(A) \rightarrow$ $A / \mathrm{P}(A) \rightarrow 0$ be an exact sequence.
Since $\mathrm{P}$ be an idempotent radical $\Rightarrow \mathrm{P}(A) \in \mathrm{T}_{\mathrm{P}}$.

$(2) \Rightarrow(3)$. It is obvious.

$(3) \Longrightarrow(4)$. Since there is an exact sequence $0 \rightarrow$ $T \rightarrow A \rightarrow F \rightarrow 0$,

Then $\mathrm{P}(\mathrm{P}(A))=\mathrm{P}(T)=T=\mathrm{P}(A) \Rightarrow \mathrm{P} \quad$ is $\quad$ an idempotent preradical with $T \in \mathrm{T}_{\mathrm{P}}$.

Also $F=A / \mathrm{P}(A) \in \mathrm{F}_{\mathrm{P}} \Rightarrow \mathrm{P}(F)=0 \Rightarrow$

$\mathrm{P}(A / \mathrm{P}(A))=0 \Longrightarrow \mathrm{P}$ is a radical.

$(4) \Rightarrow(1)$. It is obvious.

\section{Conflicts of Interest: None.}

\section{Reference:}

1. Al-thani HM. Projective and Injective Semimodules over Semirings. PhD[dissertation]. East London University; 1998.

2. Bican L, Jambor P, Kepka T, Nemec P. Preradicals. Comment Math Univ Carolinae. 1974;15(1):75-83.

3. Tsiba JR, Sow D. On Generators and Projective Semimodules. Int J Algebra. 2010Sep;4(24):11531167.

4. Chaudhari JN, Bonde DR. On Exact Sequence of Semimodules over Semirings. ISRN Algebra. 2013;15.

5. Katsov Y, Nam TG, Tuyen NX. On Subtractive Semisimple Semirings. Algebra Colloquium. 2009Sep;16(3)415-426.

6. Cekena S, Alkana M, Smithp PA. The Dual Notion of the Prime Radical of a Module. J Algebra. 2013; 3(92) 265-275.

7. Farzalipour F, Ghiasv P. Generalization of Semiprime Subsemimodule. J Bangladesh Acad. Sci. 2016;40(2)187-197.

8. Tuyen NX, H. Thang HX. On Superfluous Subsemimodules. Georgian Math J. 2003;10(4)76377.

9. Pawar K. A Note on Essential Subsemimodules. New Trends Math Sciences. 2013;1(2)18-21.

10. Alhossaini AM, Aljebory ZA. Fully Dual Stable Semimodules. JIKhS. 2017;1(1)92-100.

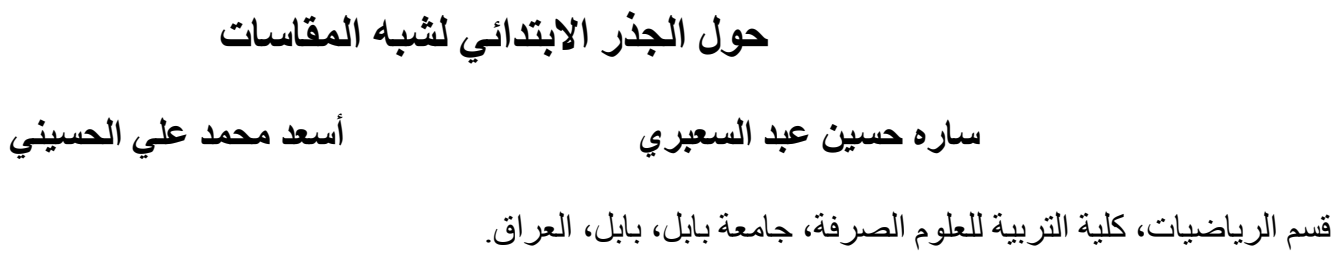

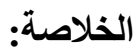

في هذا البحث درسنا مفهوم الجذر الابندائي على بعض الأصناف الجزئية من صنف شبه المقاسات مع تثناكلات شبه المقاسات

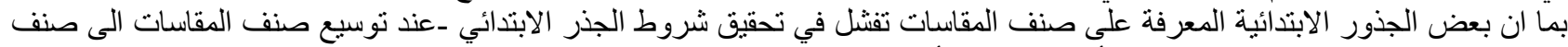

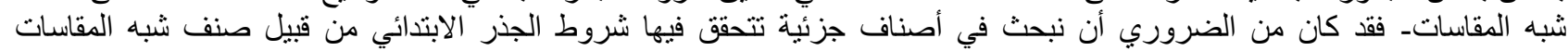

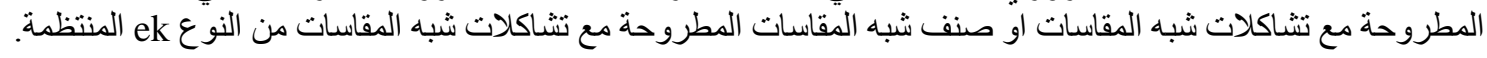
الكلمات المفتاحية: الجذر الابتدائي، شبه المقاس، شبه مقاس -Ş مطروح، تشاكل منتظم-ek 